Ernährung 2007 · 1:443

DOI 10.1007/s12082-007-0114-3

(C) Springer Gesundheits- und

Pharmazieverlag 2007

\author{
S. Fankhänel \\ Chefredakteurin „Ernährung - Wissenschaft und Praxis"
}

\title{
Die Bedeutung der Zeit
}

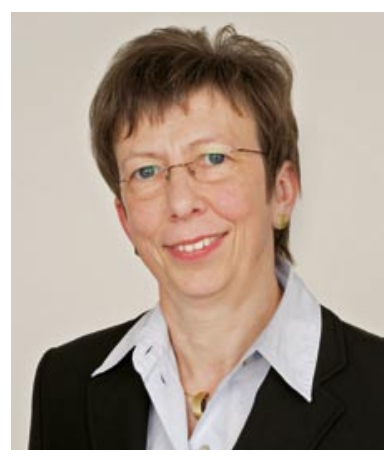

Die innere Uhr des Menschen steht im Mittelpunkt dieser Ausgabe der „ErnährungWissenschaft und Praxis“. Im Tagesverlauf rhythmische Schwankungen körperlicher Funktionen sind ein Grundphänomen des Lebens. Allerdings werden sie uns meist nur bewusst, wenn beispielsweise bei einem Jetlag oder Schichtarbeit die innere Uhr aus dem Gleichgewicht gerät. Wo und wie aber wird der Tag-Nacht-Rhythmus gesteuert? Antworten auf diese Fragen gibt Henrik Oster, der die molekularen Grundlagen des zirkadianen Systems aufS. 451 ff. erläutert. Mit den praktischen Auswirkungen eines geänderten Tag-Nacht-Rhythmus befasst sich der Beitrag auf S. $454 \mathrm{ff}$. Die Autorinnen analysieren die Gesundheits- und Ernährungssituation bei Schichtarbeit und entwickeln daraus Vorschläge für eine bedarfsgerechte Ernährung mit beispielhaften Tagesplänen. Wie ein „Ernährungscoaching "für Schichtarbeiter aussehen kann und sich von einer „normalen“ Ernährungsberatung unterscheidet, erfahren Sie in dem Interview mit Gerta van Oost ab S. 462.

Neben den Beiträgen zum Leitthema "Ernährung und zirkadianer Rhythmus" finden Sie in dieser Ausgabe einen weiteren Beitrag zu EsKiMo (Ernährungsstudie als KiGGS-Modul). Wurde bisher ver- mutet, dass die meisten Jugendlichen in Deutschland weniger trinken als empfohlen, zeigen repräsentative Daten aus EsKiMo, dass die 12- bis 17-Jährigen im Durchschnitt ausreichend Flüssigkeit aufnehmen (S. 444 ff.). Erfreulicherweise greifen die Befragten hauptsächlich zu Mineral- bzw. Leitungswasser als Durstlöscher. Allerdings trinken die über 15-jährigen männlichen Jugendlichen große Mengen Softdrinks. Beobachtet wurde außerdem, dass die älteren männlichen Jugendlichen in unerwünscht hohem Maße alkoholische Getränke konsumieren. Die große Bedeutung der richtigen Getränkeauswahl im Kindesalter zeigt auch die Studie „Trinkfit - macht mit!“. Erste Ergebnisse daraus finden Sie in der Rubrik „Ernährung aktuell“ auf S. 476.

Im November veröffentlichte der World Cancer Research Fund (WCRF) gemeinsam mit dem American Institute for Cancer Research (AICR) den zweiten Expertenbericht „Food, Nutrition, Physical Activity, and the Prevention of Cancer: a Global Perspective". Seit dem ersten Bericht, der 1997 erschien, wurden zahlreiche neue wissenschaftliche Erkenntnisse zur Krebsprävention publiziert. Daher begannen WCRF und AICR 2001 mit der Erstellung des nun vorgelegten zweiten Reports. Die Kernaussagen sowie die Empfehlungen für die Krebsprävention stellen wir Ihnen ab S. 464 vor. Lesen Sie außerdem das Interview mit Claus Leitzmann, der als Mitglied des Expertenausschusses wesentlich an der Erstellung des Leitfadens mitgewirkt hat.

Über neue Erkenntnisse bei der Erforschung des Bittergeschmacks berichten wir in dem Beitrag auf S. 470 ff. Die Arbeitsgruppe von Wolfgang Meyerhof am Deutschen Institut für Ernährungsforschung Potsdam-Rehbrücke hat kürzlich nachgewiesen, dass keine menschliche Bitterge- schmackszelle der anderen gleicht, und damit ein bisher umstrittenes Erklärungsmodell zur Geschmackswahrnehmung bestätigt. Einzelheiten dazu finden Sie auch in dem Interview mit Wolfgang Meyerhof.

In diesem Sinne: Genießen Sie trotz aller vorweihnachtlichen Hektik den Advent und finden Sie Zeit für sich und Ihre Familie. Die Redaktion und das gesamte Team der „Ernährung - Wissenschaft und Praxis" wünschen Ihnen frohe Weihnachten und ein gutes Neues Jahr.

Ihre

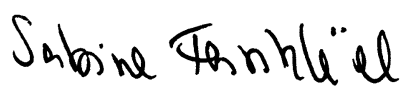

